\title{
MARKET STRUCTURE AND RIVALRY: NEW EVIDENCE WITH A NON-LINEAR MODEL
}

\author{
Michael L. Marlow, John P. Link, and Robert P. Trost*
}

\begin{abstract}
It is argued that the estimation techniques used by previous researchers to study rivalry in financial markets are inappropriate. The assumptions of both ordinary least-squares and Tobit analysis are violated when these techniques are used to analyze mobility and turnover data. To overcome the difficulties in the previous studies, we suggest a non-linear model (which is closely related to the Poisson model). This model is designed for describing frequency data and is not subject to the criticisms to which ordinary least-squares and Tobit are subject.
\end{abstract}

\section{Introduction}

Measures of mobility and turnover have recently been employed in order to test the hypothesis that the degree of rivalry is related to market structure. Heggestad and Rhoades (1976, p. 444) argue that “... a competitive market structure should force a kind of conduct or rivalry among member firms that would be reflected in a relatively large amount of mobility and turnover." Support for the hypothesis that financial market structure influences firm mobility and turnover is provided in Heggestad and Rhoades (1976), Rhoades (1980), and Rhoades and Rutz (1981). Those studies are particularly useful because of the complex problem of choosing appropriate measures of conduct from among the multitude of price and nonprice dimensions of conduct. Mobility and turnover measure the overall symptoms of competition (or lack thereof) and reflect price and nonprice dimensions of conduct.

The purpose of this paper is to argue that the estimation techniques used by previous researchers to study mobility and turnover are inappropriate. The most common estimation technique, the one used in all of the studies cited above, is ordinary least-squares (OLS). That technique is appropriate when two conditions are met: (1) the expectation of the dependent variable is equal to a linear combination of the explanatory variables, and (2) the variance of the dependent variable is constant across observations. The first condition implies that the expectation of the dependent variable would be negative for some value of the explanatory variables. However, since mobility and turnover are variables that assume only non-negative integers for their values, their expectations can never be negative. Thus condition 1 is not true of either mobility or turnover. Furthermore, it seems reasonable to assume that the variance of mo-

Received for publication February 1, 1983. Revision accepted for publication May 8, 1984.

*U.S. Department of Treasury, The George Washington University, and The George Washington University, respectively.

The authors acknowledge the constructive comments of anonymous referees. bility (or turnover) would increase with the expectation, in violation of condition 2 .

Rhoades and Rutz (1981) use Tobit analysis as an alternative to OLS and find that the results from the two techniques are generally consistent with each other. But since Tobit analysis assumes that the dependent variable is normally distributed, it does not seem to be an appropriate method to use for a discrete variable, which by its very nature cannot be so distributed. Consequently, it may not be very interesting that the two techniques generate similar results.

To overcome the difficulties in the previous studies, we suggest a non-linear model (which is closely related to the Poisson model) as a method for analyzing mobility and turnover data. This model does not have any of the inadequacies of OLS (i.e., the linear model) and Tobit. Section II summarizes the non-linear model. Section III describes the data to be analyzed. Section IV discusses the empirical results. Section V presents our conclusions.

\section{The Non-Linear Model ${ }^{1}$}

The model that we employ in order to analyze mobility and turnover data is given by three asşumptions: first, the expectation of the $i^{\text {th }}$ observation of the dependent variable, $Y_{i}$, is equal to $\exp \left(\beta^{\prime} x_{i}\right)$, where $x_{i}$ is a $K$ by 1 vector of explanatory variables and $\beta$ is a $K$ by 1 vector of fixed but unknown parameters. Second, the variance of $Y_{i}$ is proportional to the expectation. Third, the $N$ observations of the dependent variable are uncorrelated with each other. These three assumptions are summarized by the following matrix equations:

$$
\begin{aligned}
E(Y) & =\exp (X \beta)=m \\
\operatorname{cov}(Y) & =\sigma^{2} D(m)
\end{aligned}
$$

where $Y$ is the $N$ by 1 vector of observations of the dependent variable, where $X$ is the $N$ by $K$ matrix of explanatory variables that may include an intercept term, and where $D(m)$ is the $N$ by $N$ diagonal matrix with the vector of expectations, $m$, on the main diagonal.

The model described above includes the Poisson model as a special case. In the Poisson model one assumes that each $Y_{i}$ follows a Poisson distribution with parameter $m_{i}$ that is assumed to be equal to $\exp \left(\beta^{\prime} x_{i}\right)$.

\footnotetext{
${ }^{1}$ The model described here is presented in Link (1983). For a discussion of the Poisson model, see chapters 1 through 4 of Haberman (1974). The reader is encouraged to read Maddala (1983) for a more accessible introduction. See Hausman et al. (1984) for a discussion of some problems with the Poisson model.
} 
It follows that both the mean and the variance of $Y_{i}$ are equal to $m_{i}$ in the Poisson model. Thus for the Poisson model, $\sigma^{2}$ is equal to $1 .^{2}$

One method of estimation would be to use non-linear weighted least squares (NWLS). However, Link (1983) has shown that the NWLS estimate of $\beta$ is identical to the maximal-likelihood estimator for the Poisson model. Denote this estimator as $\hat{\beta}$. The correct asymptotic covariance matrix of $\hat{\beta}$ is equal to $\sigma\left[X^{\prime} D(m) X\right]^{-1}$, which is $\sigma^{2}$ times the asymptotic covariance matrix of the maximal-likelihood estimator for the Poisson model. Since $\sigma^{2}$ can be estimated by Pearson's chi-square statistic (i.e., $\left.\sum_{i}\left(Y_{i}-m_{i}\right)^{2} / m_{i}\right)$ divided by $N-K$, an easy method of obtaining the NWLS estimate of $\beta$ and its asymptotic covariance matrix presents itself. First, obtain the maximal-likelihood estimator of $\beta$ for the Poisson model. This step can be done with generalized least-squares. Second, estimate $\sigma^{2}$ by using the formula

$$
\hat{\boldsymbol{\sigma}}^{2}=\left[\sum_{i}\left(Y_{i}-m_{i}\right)^{2} / m_{i}\right] / N-K
$$

where $\hat{\sigma}^{2}$ is the estimate of $\sigma^{2}$. Third, obtain the correct asymptotic covariance matrix of $\hat{\beta}$ by using the formula

$$
\operatorname{var}(\hat{\beta})=\sigma^{2}\left(X^{\prime} D(m) X\right)^{-1}
$$

Three features of the non-linear model described above make it attractive for describing frequency data in general and mobility and turnover data in particular. First, the mean of the dependent variable is always positive. Second, the variance of the dependent variable increases with its mean (since the two are proportional to each other). Third, the computation of the NWLS estimator is almost as easy as OLS, since the NWLS estimator is identical to the maximal-likelihood estimator for the Poisson model, and that estimator can be computed by iterative generalized least-squares. In summary, the non-linear model is consistent with the restrictions on frequency data, and its non-linear weighted least-squares estimator is easy to compute.

\section{Description of the Data}

The sample consists of data on savings and loan associations for 99 SMSAs in $1979 .{ }^{3}$ The choice of SMSAs is made solely on the availability of data. The choice of variables is based on the work of Heggestad and Rhoades (1976), Rhoades (1980), and Rhoades and Rutz (1981) although those researchers all used data sets

\footnotetext{
${ }^{2}$ But note that assuming $\sigma^{2}$ to be equal to 1 does not imply the Poisson model, since there are distributions other than the Poisson that are included in the model described above and for which $\sigma^{2}$ is equal to 1 . Note also that while the Poisson model requires that the dependent variable take integer values, the more general non-linear model requires only that the expectation of the dependent variable always be positive.

${ }^{3}$ All data on savings and loan associations were obtained from Summary of Savings Accounts by Geographic Area and unpublished reports of the Federal Home Loan Bank Board.
}

for commercial banks, rather than for savings and loan associations. Each of the variables is defined at the level of the SMSA (i.e., for each SMSA there is one observation of each of the variables).

Three different measures of rivalry are employed as the dependent variable. Turnover, $R_{1}$, is the number of times that firms below the top five move into the top five. The size of a firm is defined to be the average value of its deposits for the years 1976 through 1979. Mobility, $R_{2}$, is the number of rank changes that take place among the five largest firms. The variable $R_{3}$ is the sum of $R_{1}$ and $R_{2}$. That variable has been used to measure stability in Heggestad and Rhoades (1976), Rhoades (1980), and Rhoades and Rutz (1981). Those studies argue that $R_{3}$ reflects shifts among leading firms as well as encroachment on leading firms by the second echelon of firms.

In addition to a variable that is set equal to 1 for all observations, six explanatory variables are employed:

The three-firm concentration ratio, $C R$, is the collective market share (as measured by value of deposits) of the three leading firms. Higher levels of concentration, ceteris paribus, are expected to be associated with lower levels of rivalry. ${ }^{4}$

Entry, $E$, is the net number of entries divided by the number of existing firms over the period 1976-1979. ${ }^{5}$ It is expected that more entry leads to higher levels of rivalry.

The percentage change in deposits from 1976 to 1979 , $G$, is expected to exert a positive influence on rivalry for two reasons. First, rapidly expanding markets are expected to be most attractive to new entrants. Second, uncertainty about inter-firm relations may increase with market deposits as firms are uncertain of competitors' plans toward the enlarged market. ${ }^{6}$

Market size, $D$, is the total value of deposits in 1979. This variable controls for differences in the portions of markets that new or existing firms need to capture in order to affect rivalry. A positive relation between market size and rivalry is expected because larger markets may provide for both easier capture of significant market shares and a smaller volume of deposits necessary to influence rivalry. ${ }^{?}$

The variable $U B$ is set equal to 1 if there is branching and to 0 otherwise. By allowing branching, states may

\footnotetext{
${ }^{4}$ Support of this hypothesis is provided in Heggestad and Rhoades (1976), Rhoades (1980), and Rhoades and Rutz (1981).

${ }^{5}$ Two items are noted. First, entry is calculated so as to exclude increases in the numbers of branches and mergers over the period 1976-79. Second, two alternative measures of entry were considered: the number of entries, and a binary variable that measures the presence or absence of entry. Since both proved inferior to the above measure in terms of $t$-statistics, these results are not reported.

${ }^{6}$ Market growth was found to exert a positive influence on turnover in Heggestad and Rhoades (1976).

${ }^{7}$ No relation between market size and rivalry is found in Rhoades (1980).
} 
TABLE 1.-DESCRIPTION OF VARIABLES

\begin{tabular}{cl}
\hline \hline Variable & \multicolumn{1}{c}{ Description } \\
\hline$R_{1}$ & $\begin{array}{l}\text { Turnover among the five largest firms } \\
(1976-79)\end{array}$ \\
$R_{2}$ & Mobility among the five largest firms \\
& $(1976-79)$ \\
$R_{3}$ & $R_{1}+R_{2}$ \\
$C R$ & Three-firm concentration ratio (1979) \\
$E$ & Net entry (1976-79)/number of firms (1979) \\
$G$ & Percentage change in deposits (1976-79) \\
$D$ & Total deposits (1979) \\
$U B$ & Dummy for unit branching (1979) \\
$H C$ & Dummy for holding companies (1979) \\
\hline
\end{tabular}

provide for easier entry and thereby encourage higher levels of rivalry. ${ }^{8}$

The variable $H C$ is set equal to 1 if holding companies are present and to 0 otherwise. This holding-company variable is included because holding companies perform many of the same functions as branching. ${ }^{9}$

Table 1 summarizes the variables used in this study.

\section{Empirical Results}

Table 2 displays the NWLS estimates of the model described in section II. ${ }^{10}$ Coefficients on the concentration variable are statistically significant and negative in the cases of mobility and the sum of mobility and turnover. That is consistent with expectations. Also consistent with expectations is the positive sign of the entry variable, $E$. However, it is statistically significant in only the case of turnover. No significant relation between market growth, $G$, and rivalry is observed. Market size, $D$, is not found to exert a significant

\footnotetext{
${ }^{8}$ Rhoades (1980) and Rhoades and Rutz (1981) fail to find any support for this hypothesis. Heggestad and Rhoades (1976) provide weak support for this hypothesis in the case of mobility.

${ }^{9}$ Heggestad and Rhoades (1976) and Rhoades and Rutz (1981) find that the number of holding companies positively affects rivalry while Rhoades (1980) finds that the presence of holding companies positively affects rivalry.

${ }^{10}$ Both the turnover variable, $R_{1}$, and the mobility variable, $R_{2}$, have upper bounds as well as lower bounds of zero. As long as an SMSA has at least $10 \mathrm{~S} \& \mathrm{Ls}$, its turnover variable has an upper bound of five. So that the upper bound on the turnover variable would be the same for all SMSAs, we have deleted from the sample all SMSAs with fewer than ten S \& Ls. In this way our original sample of 152 was reduced to 99 . The mobility variable has an upper bound that ranges from 28 for an SMSA with $10 \mathrm{~S} \&$ Ls to 940 for an SMSA with 193 S \& Ls. The maximal observed value for turnover was 3 while the maximal observed value for mobility was 13 . Since neither turnover nor mobility ever came close to its upper bound, we expect that the presence of the upper bounds (for which the non-linear model makes no provision) should make little difference in our results.
}

TABle 2. - NWLS Estimates of the Non-Linear Model ( $t$-statistics are shown below estimated coefficients)

\begin{tabular}{lccc}
\hline \hline & Turnover & Mobility \\
& $R_{1}$ & $R_{2}$ & $\begin{array}{c}\text { Turnover }+ \text { Mobility } \\
R_{1}+R_{2}\end{array}$ \\
\hline Concentration & -0.002 & $-0.02^{\mathrm{b}}$ & $-0.01^{\mathrm{a}}$ \\
& $(0.18)$ & $(-1.90)$ & $(1.58)$ \\
Entry $E$ & $0.24^{\mathrm{a}}$ & 0.16 & 0.19 \\
& $(1.46)$ & $(1.01)$ & $(1.28)$ \\
Growth $G$ & 0.02 & 0.11 & 0.06 \\
& $(0.39)$ & $(0.68)$ & $(0.81)$ \\
Size $D$ & -0.02 & -0.05 & -0.04 \\
& $(-0.42)$ & $(-1.07)$ & $(-0.98)$ \\
Unit Branch & 0.09 & -2.38 & -1.12 \\
$U B$ & $(0.08)$ & $(-0.71)$ & $(-0.63)$ \\
Holding Co. & 0.39 & $0.61^{\mathrm{b}}$ & $0.56^{\mathrm{b}}$ \\
$H C$ & $(1.10)$ & $(1.83)$ & $(1.76)$ \\
Constant & $0.84^{\mathrm{a}}$ & $1.14^{\mathrm{b}}$ & $1.24^{\mathrm{b}}$ \\
& $(-1.29)$ & $(1.93)$ & $(2.19)$ \\
\hline
\end{tabular}

${ }^{\text {a }}$ Significant at the 0.10 level.

${ }^{b}$ Significant at the 0.05 level.

TABle 3.-OLS Estimates of THE Linear Model ( $t$-statistics ${ }^{\mathrm{d}}$ are shown below estimated coefficients)

\begin{tabular}{lccc}
\hline \hline & $\begin{array}{c}\text { Turnover } \\
R_{1}\end{array}$ & $\begin{array}{c}\text { Mobility } \\
R_{2}\end{array}$ & $\begin{array}{c}\text { Turnover }+ \text { Mobility } \\
R_{1}+R_{2}\end{array}$ \\
\hline Concentration & -0.001 & $-0.03^{\mathrm{b}}$ & $-0.03^{\mathrm{a}}$ \\
& $(-0.25)$ & $(-1.89)$ & $(-1.58)$ \\
Entry $E$ & $0.26^{\mathrm{a}}$ & $0.76^{\mathrm{a}}$ & $1.01^{\mathrm{a}}$ \\
& $(1.62)$ & $(1.48)$ & $(1.59)$ \\
Growth & 0.02 & 0.10 & 0.12 \\
& $(0.56)$ & $(0.86)$ & $(0.83)$ \\
Size $D$ & -0.01 & -0.08 & -0.08 \\
& $(-0.40)$ & $(-1.23)$ & $(-1.08)$ \\
Unit Branch & 0.05 & -1.22 & -1.17 \\
$U B$ & $(0.09)$ & $(-0.68)$ & $(-0.53)$ \\
Holding Co. & 0.17 & $0.99^{\mathrm{b}}$ & $1.17^{\mathrm{b}}$ \\
$H C$ & $(1.01)$ & $(1.80)$ & $(1.69)$ \\
Constant & 0.45 & $3.02^{\mathrm{c}}$ & $3.48^{\mathrm{b}}$ \\
\multicolumn{1}{c}{$F$} & $(0.05)$ & $(2.82)$ & $(1.69)$ \\
\multicolumn{1}{c}{$R^{2}$} & 0.09 & 1.89 & 1.7 \\
& 0.05 & $(0.11)$ & 0.10 \\
\hline
\end{tabular}

${ }^{a}$ Significant at the 0.10 level

${ }^{b}$ Significant at the 0.05 level.

c Significant at the 0.01 level.

dAs explained in the text, there is no reason to believe that these significance levels are correct.

influence on rivalry. While the sign of its coefficient is inconsistent with expectations, it should be noted that the one previous study that included that variable (Rhoades (1980)) did not find its coefficient to be significant, although it also estimated its sign to be negative. Coefficients on the unit-branching variable, $U B$, are always statistically insignificant. In the cases of mobility and the sum of turnover and mobility, the presence of holding companies, $H C$, exerts a significant and positive effect on rivalry. 
For purposes of comparison, table 3 displays OLS estimates of the (inappropriate) linear model. ${ }^{11}$ In almost all cases, the signs on the estimated coefficients are identical to those of NWLS. However, differences do appear when one considers levels of statistical significance. In the case of concentration, both estimation techniques generate statistically significant coefficients for mobility and the sum of mobility and turnover. However, the coefficients of the NWLS estimates always exhibit lower levels. The NWLS coefficients on entry, $E$, exhibit statistical significance for only the case of turnover. The OLS coefficients on $E$ are always statistically significant. In the cases of market growth, $G$, unit branching, $U B$, and market size, $D$, neither estimation technique generates coefficients that are statistically significant. Except for turnover, both estimation techniques produce statistically significant coefficients in the case of holding-company presence, $H C$.

In making comparisons of statistical significance, one must remember that the significance levels for the OLS estimates are derived under the assumption that conditions 1 and 2 stated in section I are both correct. Since condition 1 is certainly false and condition 2 is probably false, there is no reason to believe that the significance levels reported for the OLS estimates are correct.

One must also remember that the coefficients in the non-linear model represent the percentage change in the mean of the dependent variable induced by a unit change in the explanatory variables, while the coefficients in the linear model represent the absolute change induced by a unit change. ${ }^{12}$ Hence, no simple comparison of the relative magnitudes of the estimates is possible.

In order to compare the estimates of the non-linear model with those of the linear model, we have computed, for the non-linear model, the estimated partial derivative of the expectation of the dependent variable with respect to the concentration variable. The calculation was performed where all of the variables other than

\begin{tabular}{|c|c|c|}
\hline & Mobility & $\begin{array}{l}\text { Mobility } \\
+ \text { Turnover }\end{array}$ \\
\hline Heggestad and Rhoades (1976) & -.02 & -.03 \\
\hline Rhoades (1980) & -.02 & -.04 \\
\hline Rhoades and Rutz (1981) & -.03 & -.03 \\
\hline \multicolumn{3}{|l|}{ Results from tables 2 and 3} \\
\hline Ordinary Least-Squares & -.03 & -.03 \\
\hline NWLS & -.02 & -.01 \\
\hline
\end{tabular}

This may suggest that previous work has overestimated the effects of market structure on rivalry. However, differences in coefficient size between previous and present studies may stem from differences in data sets (time periods and/or commercial banks vs. savings and loan associations).

${ }^{12}$ Note that in the non-linear model the partial derivative of $m_{i}$ with respect to $x_{i j}$ is $\beta_{j} m_{i}$, while in the linear model it is $\beta_{j}$.
TABle 4. - COMParisons of the Estimates of the Partial Derivatives OF THE EXPECTATION OF EACH RIVALRY VARIABLE WITH RESPECT TO THE CONCENTRATION VARIABLE

\begin{tabular}{lcccc}
\hline \hline & \multicolumn{3}{c}{ Non-Linear } & \\
\cline { 2 - 4 } & Min & Mean & Max & Linear \\
\hline Turnover $R_{1}$ & -.0012 & -.0011 & -.0010 & -.001 \\
Mobility $R_{2}$ & -.0834 & -.0396 & -.0194 & -.03 \\
Mobility + Turnover & & & & \\
$\quad R_{1}+R_{2}$ & -.0366 & -.0252 & -.0176 & -.03 \\
\hline
\end{tabular}

$C R$ are equal to their sample means, and where the variable $C R$ is equal to its sample minimum, maximum, and mean. Table 4 displays the results of our calculations and a comparison with the estimates of the linear model.

Two features of table 4 should be noted. First, the NWLS estimates of the partial derivatives of the expectation of each rivalry variable with respect to the concentration variable decline in absolute value as one reads from left to right. That is a consequence of two facts: (1) the partial derivative of the expectation of the rivalry variable with respect to the concentration variable is equal to the coefficient associated with the concentration variable times the expectation of the rivalry variable and (2) the expectation of the rivalry variable declines as one reads from left to right (this being a consequence of the fact that the sign of the estimated coefficient is negative in all cases).

Second, in the cases of mobility and the sum of mobility and turnover, the estimate obtained for the linear model is close to the intermediate estimate for the non-linear model. That is to be expected, since the linear model constrains the derivative to be constant, while the non-linear model constrains it to vary monotonically.

\section{Conclusion}

This study suggests a new technique to study rivalry in financial markets. A non-linear model is argued to be more appropriate than a linear model when the dependent variables are non-negative. The non-linear model is therefore more appropriate than the linear model in the case of mobility and turnover data. It is also argued that the non-linear model is more appropriate than Tobit analysis because the Tobit method assumes that the dependent variable is normally distributed.

Using measures of mobility and turnover in the savings and loan association industry, the non-linear model finds that concentration, entry, and holding company variables significantly influence mobility and the sum of mobility and turnover. Concentration is never found to explain a significant amount of the variation in turnover. 


\section{REFERENCES}

Federal Home Loan Bank Board, Summary of Savings Accounts by Geographic Area, appropriate years.

Haberman, Shelby J., The Analysis of Frequency Data (Chicago: The University of Chicago Press, 1974).

Hausman, Jerry A., Bronwyn Hall, and Zvi Griliches, "Econometric Models for Count Data with an Application to Patents-R \& D Relationship," Econometrica 52 (Jul:1984), 909-938.

$\rightarrow$ Heggestad, Arnold A., and Stephen A. Rhoades, "Concentration and Firm Stability in Commercial Banking," this
REVIEW 58 (Nov. 1976), 443-452.

Link, J. P., “A Flexible Model for Frequency Data," Economics Discussion Paper D-8220 of The George Washington University, March 1983.

Maddala, G. S., Limited-dependent and Qualitative Variables in Econometrics (Cambridge: Cambridge University Press, 1983).

Rhoades, Stephen A., "Entry and Competition in Banking," Journal of Banking and Finance 4 (1980), 143-150.

Rhoades, Stephen A., and Roger D. Rutz, "A Reexamination and Extension of the Relationship between Concentration and Firm Rank Stability," this REVIEw 63 (Aug. 1981), 446-551. 